\title{
Evaluation of Germplasm Accessions for Drought Tolerance in Green gram [Vigna radiata (L.)]
}

\author{
M. S. P. Kanavi ${ }^{1 *}$, G. Somu ${ }^{2}$, N. Marappa ${ }^{3}$, S. Rangaiah $^{3}$ and Prakash Koler
}

${ }^{1}$ Department of Genetics and Plant Breeding, College of Agriculture, Hassan, University of

Agricultural Sciences, Bangalore, Karnataka (572 225), India

${ }^{2}$ Assistant Breeder, AICRP on Sorghum, Chamarajanagara, University of Agricultural

Sciences, Bangalore, Karnataka (571 127), India

${ }^{3}$ Department of Genetics and Plant Breeding, College of Agriculture, G.K.V.K, University of

Agricultural Sciences, Bangalore, Karnataka (560 065), India

${ }^{4}$ Department Crop Physiology, College of Agriculture, Hassan, University of Agricultural

Sciences, Bangalore, Karnataka (572 225), India

\section{*Corresponding author}

\section{A B S T R A C T}

An experiment was conducted to screen 200 germplasm accessions of green gram for drought tolerance using augmented design during summer

\section{Keywords}

Green gram germplasm, GCV, PCV and Heritability

\section{Article Info}

Accepted: 15 February 2020 Available Online: 10 March 2020 2015 under drought stress condition. ANOVA revealed high significant differences among germplasm accessions for yield, yield component traits and also for drought tolerance traits.Mean squares attributable to 'Genotypes vs check entries' were significant for all the traits except seeds per podand relative water content. Genotypic coefficient of variation and phenotypic coefficient of variation were found to be on higher side for grain yield, yield components such as clusters per plant, pods per cluster and pods per plant. Higher values of GCV and PCV were also observed for drought tolerance traits such as chlorophyll content (spad chlorophyll meter reading), leaf water potential, proline content, relative water content and specific leaf area. The grain yield, yield component traits and drought tolerance traits exhibited high heritability (broad sense) coupled with high to moderate expected GAM.

\section{Introduction}

Among pulse crops, green gram is an important annual legume grown principally for its high protein seeds that are used as human food (Singh et al., 1988). The wild mung bean progenitor of present day cultivated species is widely distributed in the 
Godavari and Krishna river belts of south India and in the foothills of western Himalaya of Eastern India (Fuller, 2007; Smartt, 1990).Important beneficial factors making the green gram crop popular are; short duration (90-120 days), nitrogen fixing ability, inhibition of soil erosion, soil enrichment, low input requirements and wide adaptability.

Despite holding such a great promise, mung bean is often grown in mostly rain-fed marginal lands with limited inputs making it prone to a number of abiotic stresses. Among these stresses, drought is the major stress leading to heavy crop loss. Water is required for almost every aspect of plant growth and metabolism and its shortage affects various physiological and biochemical processes (Ahmad et al., 2014).

Soil moisture deficit is a multidimensional stress affecting plants at various levels of their organization (Yordanov, 2000). Beebe et al., (2013) reported that adaptation to drought, encompasses diverse mechanisms that enable plants to survive and produce in periods of drought stress. Single trait taken alone cannot be a good indicator of drought, since a number of traits jointly influence yield under drought stress (Kao et al., 1994).

Green gram is reported to be more susceptible to water deficits than any other grain legumes. Reduction in crop photosynthesis is caused by reduction in plants leaf leading to dry matter accumulation (Pandey et al., 1984). Siddiqui et al., (2007) reported that pod setting stage and late flowering traits appear to be the most sensitive stages to soil moisture stress and yield.

Various physiological processes associated with growth, development, and economic yield of a crop are affected due to water stress (Allahmoradi et al., 2011).Breeding efficiency for drought tolerance heavily relies on need to identify the specific physiological, biochemical and genetically controlled traits that may improve yields under drought stress condition.

So far, the drought stress has not been properly dissected into its different components at physiological, bio-chemical, genetic and molecular genetics levels. Only limited information is available in pulse crops, placing constraints in designing an appropriate breeding methodology to facilitate genetic improvement.

\section{Materials and Methods}

The experiment was conducted at experimental plot of College of Agriculture, Hassan, University of Agricultural Sciences, Bangalore. The experimental site is geographically located at Southern Transitional Zone (Zone-7) of Karnataka with an altitude of $827 \mathrm{~m}$ above Mean Sea Level (MSL) and at $33^{\prime} \mathrm{N}$ latitude and $75^{\circ} 33^{\prime}$ to $76^{\circ}$ E38' longitude. The study material consisted of 200 germplasm accessions collected from different research institutions / organizations representing different agro-climatic zones. List of germplasm accessions used in the study with their source is given in table No1.

\section{Layout of the experiment}

The experiment was conducted in an Augmented Randomized Complete Block Design with 200 germplasm accessions and 5 check varieties. As per the augmented RCBD, the check entries were replicated twice randomly in each block. There were 5 blocks, each block had 5 plots of size $3 \times 3 \mathrm{~m}^{2}$ thus each block size was $15 \mathrm{~m}^{2}$. The gross area of experimental plot was $75 \mathrm{~m}^{2}$. The row spacing was $30 \mathrm{~cm}$ and inter plant distance was $10 \mathrm{~cm}$. The experiment was conducted during summer 2015. Recommended crop production practices were followed during the crop growth period to raise healthy crop. 


\section{Imposing drought condition}

Drought condition was imposed by withholding irrigation 25 days after sowing (Baroowa and Gogoi, 2015; Pooja et al., 2019). Since the experiment was conducted during summer season, there were no unpredicted rains during the entire cropping period hence the drought condition was effectively imposed. The rainfall data of experimental site during the cropping period is given in table No.2.

\section{Plant sampling and data collection}

Observations were recorded on five randomly chosen competitive plants from each germplasm accession for all the characters except days to 50 per cent flowering and days to maturity, which were recorded on plot basis. The values of five competitive plants were averaged and expressed as mean of the respective characters. The observations were taken on the traits like; Days to $50 \%$ flowering, Days to maturity, Plant height (cm), Clusters per plant, Pods per cluster, Pods per plant, Pod length $(\mathrm{cm})$, Seeds per pod, test weight, Threshing \%, Harvest index (\%),SCMR (SPAD Chlorophyll meter reading), Leaf water potential(Mpa), Proline content $\left(\mu \mathrm{g} \mathrm{g}^{-1}\right)$, Relative water content, Specific leaf area and Seed yield per plant

\section{Results and Discussion}

\section{Assessment of genetic variability for grain yield and its component traits}

For successful crop improvement programmes, breeders need to define and assemble the required genetic variability and select for yield indirectly through yield associated and highly heritable characters (Mather, 1949). Selection is only effective if the trait has high heritability otherwise attempts to improve character through selection will be futile.

\section{Analysis of variance}

Analysis of variance revealed highly significant mean squares attributable to germplasm accessions for all the traits. Significant mean squares were recorded for all the traits. (Table 3). Mean squares attributable to 'Genotypes vs check entries' were significant for all the traits except seeds per podand relative water content. These results suggest significant differences among the germplasm accessions. The germplasm accessions as group differed significantly for all of the traits under investigation, similarly, check entries as group differed significantly for most the traits under study.

\section{Descriptive statistics for yield parameters}

Genetic variability is a pre-requisite for quantifying variability and assessing relative contribution of genetic and non-genetic sources on the quantitative traits which is useful in formulating appropriate selection strategies. The mean and range values do not reflect the total variability present in the material.

Hence, actual variance has to be estimated for the traits to know the extent of variability existed in them. The absolute values of phenotypic and genotypic variance can't be used for making comparison of degree of variability across characters as they the traits differ in their units of measurement. Hence, co-efficient of variation (PCV and GCV) which are free from measurement units are used for making comparison. Higher values for these parameters indicate large variability and vice versa.

The values of different descriptive statistics in given in table4. Days to 50 per cent flowering varied from 33.00 days to 60.00 days with a 
mean of 41.61days. Days to maturity varied from 60.00 days to 81.00 days with a mean of 69.14 days. Plant height ranged from 19.18 $\mathrm{cm}$ to $58.57 \mathrm{~cm}$ with mean value of $37.74 \mathrm{~cm}$. Values of cluster per plantvaried from 1.53 to 8.25 with mean value of 5.00. Minimum value of 1.75 and maximum value of 4.50 with mean value of 3.23 was observed for the trait pods per cluster. Pods per plant had range of values from 4.38 to 35.72 with mean value of 16.81. Pod length varied from 4.05 to 7.67 with mean value of 5.83. Seeds per pod ranged from 3.07 to 9.70 with a mean of 6.63 .

Minimum and maximum values for the trait test weight were $1.71 \mathrm{gms}$ and $5.49 \mathrm{gms}$ respectively with mean value of 3.45 gms. Threshing percentage varied from 42.89 per cent to 76.93 per cent with mean value of 62.03 per cent. Minimum value of 20.52 and maximum value of 55.95 with mean value of 35.11 was observed for the trait harvest index. Spad chlorophyll meter reading had range of values from 36.59 to 87.41 with mean value of 55.55. Leaf water potential varied from 8.14 Mpa to -2.16 Mpa with mean value of 5.74. Proline content values ranged from $62.70\left(\mu \mathrm{gg}^{-1}\right)$ to $201.33\left(\mu \mathrm{g} \mathrm{g}^{-1}\right)$ with mean value of $120.98\left(\mu \mathrm{g} \mathrm{g}^{-1}\right)$. Relative water content recorded lowest value of 33.63 and highest value of 97.18 with a mean of 68.59 . Specific leaf area had a minimum value of 31.96 and maximum of 298.29 with mean value of 156.52. Seed yield per plantranged from $0.74 \mathrm{gms}$ to $11.05 \mathrm{gms}$ with a mean value of 4.02 gms. The estimates of standardized range across traits provide clues about the occurrence of genotypes with extreme expression.

The standardized range were relatively higher for all the quantitative traits such as; plant height (1.04), cluster per plant(1.34), pods per cluster(0.85), pods per plant (1.87), pod length (0.62), seeds per pod (1.00) test weight (1.10), threshing percentage (0.55), harvest index (1.01), spad chlorophyll meter reading (0.91), leaf water potential (-1.04), proline content (1.15), relative water content (0.93), specific leaf area (1.70) and seed yield per plant (2.56) except for days to $50 \%$ flowering (0.48) and days to maturity (0.30). Higher ranges for plant height and other traits in green gram are reported by Muthuswamy et al., (2019).

Table.1 List of germplasm accessions used in the study and their source

\begin{tabular}{|c|c|c|}
\hline SI. No. & Germplasm & Location \\
\hline $\mathbf{1}$ & KM13-16 & ARS, Bidar \\
\hline $\mathbf{2}$ & KM13-19 & ARS, Bidar \\
\hline $\mathbf{3}$ & KM13-39 & ARS, Bidar \\
\hline $\mathbf{4}$ & GG13-7 & ARS, Bidar \\
\hline $\mathbf{5}$ & GG13-6 & ARS, Bidar \\
\hline $\mathbf{6}$ & KM13-44 & ARS, Bidar \\
\hline $\mathbf{7}$ & GG13-10 & ARS, Bidar \\
\hline $\mathbf{8}$ & SML-668 & ARS, Bidar \\
\hline $\mathbf{9}$ & KM13-9 & ARS, Bidar \\
\hline $\mathbf{1 0}$ & IPM99-125 & ARS, Bidar \\
\hline $\mathbf{1 1}$ & LGG-596 & RARS, Guntur \\
\hline $\mathbf{1 2}$ & LGG-572 & RARS, Guntur \\
\hline $\mathbf{1 3}$ & LGG-450 & RARS, Guntur \\
\hline
\end{tabular}




\begin{tabular}{|c|c|c|}
\hline 14 & LGG-583 & RARS, Guntur \\
\hline 15 & LGG-590 & RARS, Guntur \\
\hline 16 & LGG-588 & RARS, Guntur \\
\hline 17 & LGG-589 & RARS, Guntur \\
\hline 18 & LGG-579 & RARS, Guntur \\
\hline 19 & LGG-562 & RARS, Guntur \\
\hline 20 & LGG-582 & RARS, Guntur \\
\hline 21 & LGG-585 & RARS, Guntur \\
\hline 22 & AKL-170 & NBPGR, Akola \\
\hline 23 & PLM-110 & UAS, Bangalore \\
\hline 24 & LGG-577 & RARS, Guntur \\
\hline 25 & IC-436624 & IIPR, Kanpur \\
\hline 26 & IC-436723 & IIPR, Kanpur \\
\hline 27 & IC-413316 & IIPR, Kanpur \\
\hline 28 & IC-436746 & IIPR, Kanpur \\
\hline 29 & VGG10-010 & TNAU, Coimbatore \\
\hline 30 & VGG04-011 & TNAU, Coimbatore \\
\hline 31 & VGG04-007 & TNAU, Coimbatore \\
\hline 32 & COGG-93 & TNAU, Coimbatore \\
\hline 33 & VBNGG-2 & TNAU, Coimbatore \\
\hline 34 & TARM-2013 & TNAU, Coimbatore \\
\hline 35 & VGG04-005 & TNAU, Coimbatore \\
\hline 36 & COGG-920 & TNAU, Coimbatore \\
\hline 37 & VGG07-003 & TNAU, Coimbatore \\
\hline 38 & VGG10-002 & TNAU, Coimbatore \\
\hline 39 & VGG-112 & TNAU, Coimbatore \\
\hline 40 & IC-92048 & NBPGR, Akola \\
\hline 41 & AKL-103 & NBPGR, Akola \\
\hline 42 & AKL-39 & NBPGR, Akola \\
\hline 43 & AKL-106 & NBPGR, Akola \\
\hline 44 & AKL-225 & NBPGR, Akola \\
\hline 45 & AKL-95 & NBPGR, Akola \\
\hline 46 & AKL-194 & NBPGR, Akola \\
\hline 47 & AKL-212 & NBPGR, Akola \\
\hline 48 & AKL-195 & NBPGR, Akola \\
\hline 49 & AKL-211 & NBPGR, Akola \\
\hline 50 & KM13-11 & ARS, Bidar \\
\hline 51 & KM13-30 & ARS, Bidar \\
\hline 52 & KM13-45 & ARS, Bidar \\
\hline 53 & KM13-18 & ARS, Bidar \\
\hline 54 & KM13-5 & ARS, Bidar \\
\hline 55 & KM13-02 & ARS, Bidar \\
\hline 56 & KM13-37 & ARS, Bidar \\
\hline
\end{tabular}




\begin{tabular}{|c|c|c|}
\hline 57 & KM13-23 & ARS, Bidar \\
\hline 58 & KM13-55 & ARS, Bidar \\
\hline 59 & KM13-12 & ARS, Bidar \\
\hline 60 & GG13-9 & ARS, Bidar \\
\hline 61 & KM13-49 & ARS, Bidar \\
\hline 62 & GG13-4 & ARS, Bidar \\
\hline 63 & GG13-54 & ARS, Bidar \\
\hline 64 & KM13-20 & ARS, Bidar \\
\hline 65 & GG13-5 & ARS, Bidar \\
\hline 66 & Chinamung & ARS, Bidar \\
\hline 67 & GG13-2 & ARS, Bidar \\
\hline 68 & KM13-26 & ARS, Bidar \\
\hline 69 & KM13-47 & ARS, Bidar \\
\hline 70 & KM13-41 & ARS, Bidar \\
\hline 71 & KM13-11 & ARS, Bidar \\
\hline 72 & KM13-42 & ARS, Bidar \\
\hline 73 & GG13-11 & ARS, Bidar \\
\hline 74 & GG13-8 & ARS, Bidar \\
\hline 75 & GG13-12 & ARS, Bidar \\
\hline 76 & KM13-48 & ARS, Bidar \\
\hline 77 & IPM2-3 & ARS, Bidar \\
\hline 78 & IPM2-14 & ARS, Bidar \\
\hline 79 & PDM-139 & ARS, Bidar \\
\hline 80 & LGG-580 & RARS, Guntur \\
\hline 81 & PM-112 & TNAU, Coimbatore \\
\hline 82 & LGG-578 & NBPGR, Akola \\
\hline 83 & LGG-563 & NBPGR, Akola \\
\hline 84 & LGG-594 & NBPGR, Akola \\
\hline 85 & TM-96-2 & NBPGR, Akola \\
\hline 86 & LGG-593 & NBPGR, Akola \\
\hline 87 & LGG-591 & NBPGR, Akola \\
\hline 88 & PM-115 & NBPGR, Akola \\
\hline 89 & LGG-587 & NBPGR, Akola \\
\hline 90 & PM-113 & NBPGR, Akola \\
\hline 91 & LGG-586 & NBPGR, Akola \\
\hline 92 & IC-436775 & NBPGR, Akola \\
\hline 93 & IC-413311 & NBPGR, Akola \\
\hline 94 & IC-398984 & NBPGR, Akola \\
\hline 95 & IC-436767 & NBPGR, Akola \\
\hline 96 & IC-436573 & NBPGR, Akola \\
\hline 97 & LGG-584 & NBPGR, Akola \\
\hline 98 & LGG-592 & NBPGR, Akola \\
\hline 99 & LGG-555 & NBPGR, Akola \\
\hline
\end{tabular}




\begin{tabular}{|c|c|c|}
\hline 100 & LGG-564 & NBPGR, Akola \\
\hline 101 & LGG-460 & RARS, Guntur \\
\hline 102 & LGG-595 & RARS, Guntur \\
\hline 103 & LGG-566 & RARS, Guntur \\
\hline 104 & IC-553514 & IIPR, Kanpur \\
\hline 105 & IC-413319 & IIPR, Kanpur \\
\hline 106 & IC-436542 & IIPR, Kanpur \\
\hline 107 & IC-546493 & IIPR, Kanpur \\
\hline 108 & IC-436594 & IIPR, Kanpur \\
\hline 109 & IC-436630 & IIPR, Kanpur \\
\hline 110 & IC-436668 & IIPR, Kanpur \\
\hline 111 & IC-436555 & IIPR, Kanpur \\
\hline 112 & IC-413314 & IIPR, Kanpur \\
\hline 113 & AKL-20 & NBPGR, Akola \\
\hline 114 & AKL-89 & NBPGR, Akola \\
\hline 115 & AKL-228 & NBPGR, Akola \\
\hline 116 & AKL-184 & NBPGR, Akola \\
\hline 117 & AKL-182 & NBPGR, Akola \\
\hline 118 & AKL-230 & NBPGR, Akola \\
\hline 119 & AKL-229 & NBPGR, Akola \\
\hline 120 & AKL-86 & NBPGR, Akola \\
\hline 121 & IC-436646 & IIPR, Kanpur \\
\hline 122 & IC-343964 & IIPR, Kanpur \\
\hline 123 & IC-436528 & IIPR, Kanpur \\
\hline 124 & IC-436723 & IIPR, Kanpur \\
\hline 125 & IC-546491 & IIPR, Kanpur \\
\hline 126 & IC-546481 & IIPR, Kanpur \\
\hline 127 & IC-398988 & IIPR, Kanpur \\
\hline 128 & VGG10-005 & TNAU, Coimbatore \\
\hline 129 & VBN-223 & TNAU, Coimbatore \\
\hline 130 & COGG-912 & TNAU, Coimbatore \\
\hline 131 & VBN(G9)-3 & TNAU, Coimbatore \\
\hline 132 & ML-1165 & TNAU, Coimbatore \\
\hline 133 & VGG04-025 & TNAU, Coimbatore \\
\hline 134 & VGG04-004 & TNAU, Coimbatore \\
\hline 135 & VGG04-149 & TNAU, Coimbatore \\
\hline 136 & COGG-954 & TNAU, Coimbatore \\
\hline 137 & VGG08-002 & TNAU, Coimbatore \\
\hline 138 & VBN-1 & TNAU, Coimbatore \\
\hline 139 & VGG-119 & TNAU, Coimbatore \\
\hline 140 & VC3890-A & TNAU, Coimbatore \\
\hline 141 & DGGV-4 & UAS, Raichur \\
\hline 142 & KPS-1 & UAS, Raichur \\
\hline
\end{tabular}




\begin{tabular}{|c|c|c|}
\hline $\mathbf{1 4 3}$ & CGG-973 & UAS, Raichur \\
\hline $\mathbf{1 4 4}$ & CN9-5 & UAS, Raichur \\
\hline $\mathbf{1 4 5}$ & KPS-2 & UAS, Raichur \\
\hline $\mathbf{1 4 6}$ & VC-6173 & UAS, Raichur \\
\hline $\mathbf{1 4 7}$ & VC-6368 & UAS, Raichur \\
\hline $\mathbf{1 4 8}$ & CO-6 & UAS, Raichur \\
\hline $\mathbf{1 4 9}$ & Harsha & UAS, Raichur \\
\hline $\mathbf{1 5 0}$ & PLM-92 & UAS, Bangalore \\
\hline $\mathbf{1 5 1}$ & MH-709 & UAS, Raichur \\
\hline $\mathbf{1 5 2}$ & LGG-460 & RARS, Guntur \\
\hline $\mathbf{1 5 3}$ & KGS-5 & UAS, Raichur \\
\hline $\mathbf{1 5 4}$ & Barimung-4 & UAS, Raichur \\
\hline $\mathbf{1 5 5}$ & AKL-189 & NBPGR, Akola \\
\hline $\mathbf{1 5 6}$ & AKL-168 & NBPGR, Akola \\
\hline $\mathbf{1 5 7}$ & AKL-218 & NBPGR, Akola \\
\hline $\mathbf{1 5 8}$ & AKL-179 & NBPGR, Akola \\
\hline $\mathbf{1 5 9}$ & AKL-185 & NBPGR, Akola \\
\hline $\mathbf{1 6 0}$ & AKL-163 & NBPGR, Akola \\
\hline $\mathbf{1 6 1}$ & COGG-912 & TNAU, Coimbatore \\
\hline $\mathbf{1 6 2}$ & IC-73451 & NBPGR, Akola \\
\hline $\mathbf{1 6 3}$ & IC- 105690 & NBPGR, Akola \\
\hline $\mathbf{1 6 4}$ & IC-73534 & NBPGR, Akola \\
\hline $\mathbf{1 6 5}$ & IC-73412 & NBPGR, Akola \\
\hline $\mathbf{1 6 6}$ & IC-39605 & NBPGR, Akola \\
\hline $\mathbf{1 6 7}$ & IC-73472 & NBPGR, Akola \\
\hline $\mathbf{1 6 8}$ & IC- 92053 & NBPGR, Akola \\
\hline $\mathbf{1 6 9}$ & IC-73779 & NBPGR, Akola \\
\hline $\mathbf{1 7 0}$ & IC-73462 & NBPGR, Akola \\
\hline $\mathbf{1 7 1}$ & IC- 118992 & NBPGR, Akola \\
\hline $\mathbf{1 7 2}$ & IC-53783 & NBPGR, Akola \\
\hline $\mathbf{1 7 3}$ & IC-73456 & NBPGR, Akola \\
\hline $\mathbf{1 7 4}$ & IC-73458 & NBPGR, Akola \\
\hline $\mathbf{1 7 5}$ & AKL-105 & NBPGR, Akola \\
\hline $\mathbf{1 7 6}$ & AKL-213 & NBPGR, Akola \\
\hline $\mathbf{1 7 7}$ & AKL-169 & NBPGR, Akola \\
\hline $\mathbf{1 7 8}$ & AKL-220 & NBPGR, Akola \\
\hline $\mathbf{1 7 9}$ & AKL-84 & NBPGR, Akola \\
\hline $\mathbf{1 8 0}$ & AKL-82 & NBPGR, Akola \\
\hline $\mathbf{1 8 1}$ & AKL-97 & NBPGR, Akola \\
\hline $\mathbf{1 8 2}$ & AKL-226 & NBPGR, Akola \\
\hline $\mathbf{1 8 3}$ & AKL-24 & NBPGR, Akola \\
\hline $\mathbf{1 7 0}$ & IC-73462 & NBPGR, Akola \\
\hline $\mathbf{1 7 1}$ & IC-118992 & NBPGR, Akola \\
\hline & & \\
\hline
\end{tabular}




\begin{tabular}{|l|c|l|}
\hline $\mathbf{1 7 2}$ & IC-53783 & NBPGR, Akola \\
\hline $\mathbf{1 7 3}$ & IC-73456 & NBPGR, Akola \\
\hline $\mathbf{1 7 4}$ & IC-73458 & NBPGR, Akola \\
\hline $\mathbf{1 7 5}$ & AKL-105 & NBPGR, Akola \\
\hline $\mathbf{1 7 6}$ & AKL-213 & NBPGR, Akola \\
\hline $\mathbf{1 7 7}$ & AKL-169 & NBPGR, Akola \\
\hline $\mathbf{1 7 8}$ & AKL-220 & NBPGR, Akola \\
\hline $\mathbf{1 7 9}$ & AKL-84 & NBPGR, Akola \\
\hline $\mathbf{1 8 0}$ & AKL-82 & NBPGR, Akola \\
\hline $\mathbf{1 8 1}$ & AKL-97 & NBPGR, Akola \\
\hline $\mathbf{1 8 2}$ & AKL-226 & NBPGR, Akola \\
\hline $\mathbf{1 8 3}$ & AKL-24 & NBPGR, Akola \\
\hline $\mathbf{1 8 4}$ & AKL-174 & NBPGR, Akola \\
\hline $\mathbf{1 8 5}$ & AKL-161 & NBPGR, Akola \\
\hline $\mathbf{1 8 6}$ & AKL-180 & NBPGR, Akola \\
\hline $\mathbf{1 8 7}$ & AKL-222 & NBPGR, Akola \\
\hline $\mathbf{1 8 8}$ & AKL-187 & NBPGR, Akola \\
\hline $\mathbf{1 8 9}$ & AKL-216 & NBPGR, Akola \\
\hline $\mathbf{1 9 0}$ & AKL-29 & NBPGR, Akola \\
\hline $\mathbf{1 9 1}$ & AKL-90 & NBPGR, Akola \\
\hline $\mathbf{1 9 2}$ & AKL-227 & NBPGR, Akola \\
\hline $\mathbf{1 9 3}$ & AKL-200 & NBPGR, Akola \\
\hline $\mathbf{1 9 4}$ & AKL-92 & NBPGR, Akola \\
\hline $\mathbf{1 9 5}$ & AKL-183 & NBPGR, Akola \\
\hline $\mathbf{1 9 6}$ & AKL-176 & NBPGR, Akola \\
\hline $\mathbf{1 9 7}$ & AKL-191 & NBPGR, Akola \\
\hline $\mathbf{1 9 8}$ & AKL-165 & NBPGR, Akola \\
\hline $\mathbf{1 9 9}$ & AKL-164 & NBPGR, Akola \\
\hline $\mathbf{2 0 0}$ & AKL-192 & NBPGR, Akola \\
\hline & & \\
\hline
\end{tabular}

Table.2 Meteorological data of experimental site for the year 2015

\begin{tabular}{|c|l|c|c|c|}
\hline Year & Months & Temperature $\left({ }^{\circ} \mathbf{C}\right)$ & Relative humidity $(\mathbf{\%})$ & Rainfall (mm) \\
\hline $\mathbf{2 0 1 5}$ & January & 21.32 & 61.03 & 0.59 \\
\hline & February & 23.10 & 50.72 & Nil \\
\hline & March & 25.34 & 58.70 & $2 \mathrm{~mm}(25.03 .2015)$ \\
\hline & April & 25.87 & 66.55 & Nil \\
\hline
\end{tabular}

The standardized range were relatively higher for all the quantitative traits such as; plant height (1.04), cluster per plant(1.34), pods per cluster(0.85), pods per plant (1.87), pod length (0.62), seeds per pod (1.00) test weight (1.10), threshing percentage $(0.55)$, harvest index (1.01), spad chlorophyll meter reading $(0.91)$, leaf water potential $(-1.04)$, proline content (1.15), relative water content (0.93), specific leaf area (1.70) and seed yield per plant (2.56) except for days to $50 \%$ flowering (0.48) and days to maturity (0.30). 
Table.3 Summary of augmented ANOVA for grain yield and component traits of germplasm accessions under drought condition

\begin{tabular}{|c|c|c|c|c|c|c|c|c|c|c|c|c|c|c|}
\hline Sources of Variations & DF & DFF & DM & PH & \multicolumn{2}{|c|}{ CPP } & \multicolumn{2}{|c|}{ PPC } & \multicolumn{2}{|c|}{ PPP } & \multicolumn{2}{|c|}{ PL } & SPP & TW \\
\hline Blocks (b) & 4 & $14.74 * *$ & $8.18 * * *$ & $65.31 * *$ & \multicolumn{2}{|c|}{$2.23 * *$} & \multicolumn{2}{|c|}{$0.11 *$} & \multicolumn{2}{|c|}{$25.23 * *$} & \multicolumn{2}{|c|}{$1.49 * *$} & $5.05^{* *}$ & $1.77 * *$ \\
\hline $\begin{array}{l}\text { Entries (e) } \\
(\text { Genotypes + Checks) }\end{array}$ & 204 & $* *$ & 18.0 & $84.47 * *$ & \multicolumn{2}{|c|}{$3.60 * *$} & \multicolumn{2}{|c|}{$0.51 * *$} & \multicolumn{2}{|c|}{$72.94 * *$} & \multicolumn{2}{|c|}{$0.75^{* *}$} & $2.70 * *$ & $0.35 * *$ \\
\hline Checks & 4 & $34.57 * *$ & $37.01 * *$ & $22.56 * *$ & \multicolumn{2}{|c|}{$1.40 * *$} & \multicolumn{2}{|c|}{$0.42 * *$} & \multicolumn{2}{|c|}{$12.50 * *$} & \multicolumn{2}{|c|}{$0.87 * *$} & $3.98 * *$ & $0.81 * *$ \\
\hline Genotypes & 199 & $14.215 * *$ & $15.14^{* *}$ & $85.71 * *$ & \multicolumn{2}{|c|}{$3.67 * *$} & \multicolumn{2}{|c|}{$0.51 * *$} & \multicolumn{2}{|c|}{$73.91 * *$} & \multicolumn{2}{|c|}{$0.73 * *$} & $2.69 * *$ & $0.31 * *$ \\
\hline Checks vs Genotypes & 1 & $521.64 * *$ & $513.06 * *$ & $85.01 * *$ & \multicolumn{2}{|c|}{$0.16^{* *}$} & \multicolumn{2}{|c|}{$1.45^{* *}$} & \multicolumn{2}{|c|}{$121.60 * *$} & \multicolumn{2}{|c|}{$4.52 * *$} & 0.03 & $5.42 * *$ \\
\hline Error & 16 & 1.32 & 0.74 & 0.98 & \multicolumn{2}{|c|}{0.04} & & & \multicolumn{2}{|c|}{0.98} & \multicolumn{2}{|c|}{0.009} & 0.05 & 0.05 \\
\hline Sources of Variations & DF & $\mathbf{T P}$ & HI & \multicolumn{2}{|c|}{ SCMR } & \multicolumn{2}{|c|}{ LWP } & \multicolumn{2}{|c|}{ PC } & \multicolumn{2}{|c|}{ RWC } & \multicolumn{2}{|c|}{ SLA } & SYPP \\
\hline Blocks (b) & 4 & $37.12 *$ & $247.54 * *$ & \multicolumn{2}{|c|}{$396.55 * *$} & \multicolumn{2}{|c|}{$1.17 * *$} & \multicolumn{2}{|c|}{$470.90 * *$} & & & & $7.34 *$ & $2.11 * *$ \\
\hline $\begin{array}{l}\text { Entries (e) } \\
(\text { Genotypes + Checks) }\end{array}$ & 204 & $37.20 * *$ & $54.41 *$ & 98.71 & & & & 170 & ** & 425 & & & $10 * *$ & $7.01 * *$ \\
\hline Checks & 4 & 17.09 & $64.39 *$ & 24.4 & & & & & & & & & 24.20 & $3.76 * *$ \\
\hline Genotypes & 199 & $27.67 *$ & $53.01 *$ & 79.5 & & & & 171 & $7 * *$ & 433. & & & $4.15^{* *}$ & $7.10 * *$ \\
\hline Checks vs Genotypes & 1 & $2014.79 * *$ & $293.20 * *$ & 4203.25 & & 32.5 & $* *$ & 382 & $9 * *$ & & & & $8.68 * *$ & $0.42 *$ \\
\hline Error & 16 & 9.83 & 19.57 & 31.14 & & & & & & & & & 339.95 & 0.09 \\
\hline
\end{tabular}

*Significant at $\mathrm{P}=0.05, * *$ Significant at $\mathrm{P}=0.01$ DFF : Days to 50\% flowering Pods plant-1 DM : Days to maturity PL : Pod length $(\mathrm{cm})$ PH : Plant height $(\mathrm{cm}) \quad$ SPP : Seeds per pod $\begin{array}{lll}\text { CPP : Cluster plant-1 } & \text { TW: test weight }(\mathrm{g}) & \text { PC : Proline content }(\mu \mathrm{g} \mathrm{g}-1) \\ \text { PPC : Pods cluster-1 } & \text { TP : Threshing \% RWC : Relative water content }(\%)\end{array}$

HI : Harvest index (\%) SLA : Specific leaf area SCMR : SPAD Chlorophyll meter reading SYPP : Seed yield plant-1 LWP : Leaf water potential(Mpa) PC : Proline content ( $\mu$ g g-1) TP : Threshing \% RWC : Relative water content (\%) 
Table.4 Descriptive statistics for grain yield and its component traits of germplasm accessions under drought condition

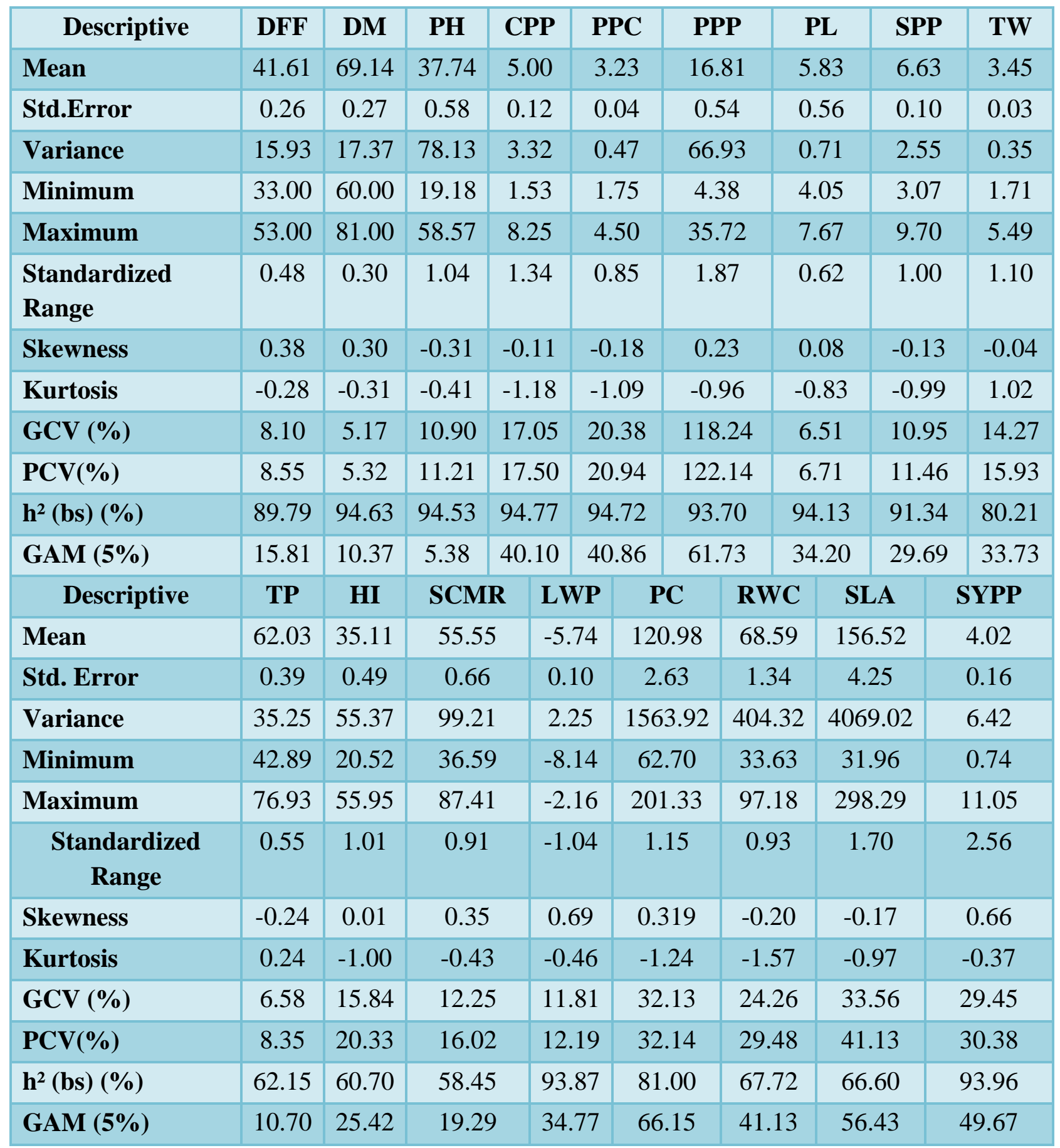

DFF : Days to $50 \%$ flowering PL : Pod length $(\mathrm{cm}) \quad$ LWP : Leaf water potential(Mpa)

DM : Days to maturity SPP : Seeds per pod PC : Proline content $(\mu \mathrm{g} g-1)$

PH : Plant height (cm) TW: test weight $(\mathrm{g}) \quad$ RWC : Relative water content (\%)

CPP : Cluster plant-1 TP : Threshing \% SLA : Specific leaf area

PPC : Pods cluster-1 HI : Harvest index (\%) SYPP : Seed yield plant-1

PPP: Pods plant-1 SCMR : SPAD Chlorophyll meter reading 
Higher ranges for plant height and other traits in green gram are reported by Muthuswamy et al., (2019). The magnitude of variation revealed by GCV and PCV in germplasm accessions were low for days to $50 \%$ flowering $(8.10 \%$ and $8.55 \%$ respectively) days to maturity $(5.17 \%$ and $5.32 \%$ respectively) threshing percentage (6.58\% and $8.35 \%$ respectively) and pod length (6.51 $\%$ and $6.71 \%$ respectively). Low GCV and PCV values indicate presence of limited variability for these traits. Moderate GCV and PCV values were observed for plant height (10.90\% and $11.21 \%$ respectively), cluster per plant $(17.05 \%$ and $17.05 \%$ respectively) harvest index (15.84 \% and $20.33 \%$ respectively), spad chlorophyll meter reading (12.25\% and $16.02 \%$ respectively) and leaf water potential $(11.81 \%$ and $12.19 \%$ respectively).

Higher standardized range (1.87) resulted in higher GCV and PCV values for pods per plant(118.24\% and $122.14 \%$ respectively). Similarly higher GCV and PCV values were observed for proline content $(31.13 \%$ and $32.14 \%$ respectively), relative water content (24.26\% and $29.48 \%$ respectively), specific leaf area (33.56\% and $41.13 \%$ respectively) and seed yield per plant $29.45 \%$ and $30.38 \%$ respectively). Muthuswamy et al., (2019) and Tejbir et al., (2009)have reported similar findings on GCV and PCV estimates.

Heritability is a quantitative measure which provides information about the relative contribution of genetic factors to the phenotypic expression. The estimates of genetic advance may be biased if phenotypic variance contains a fraction of genetic variance due to non-additive effects (Hanson et al., 1956).According to Johnson et al., (1955), heritability estimates along with genetic gain would be more useful and informative in predicting effectiveness of selection. Hence it is essential to consider the predicted genetic advance along with heritability estimates as a tool in selection for increased efficiency. Germplasm accessions exhibited relatively higher heritability for all the quantitative traits; days to $50 \%$ flowering $(89.79 \%)$, days to maturity $(94.63 \%)$, plant height $(94.53 \%)$, cluster per plant $(94.77 \%)$, pods per cluster(94.72\%), pods per plant(93.70\%), pod length $(94.13 \%)$, seeds per pod $(91.34 \%)$ test weight $(80.21 \%)$, threshing percentage $(62.15 \%)$, harvest index (60.70 \%), spad chlorophyll meter reading (58.45\%), leaf water potential (99.96\%), proline content $(81.00 \%)$, relative water content $(67.72 \%)$, specific leaf area (66.60 $\%)$ and seed yield per plant $(93.96 \%)$. Kate et al., (2017) has reported high heritability coupled with moderate genetic advance for the traits; plant height, days to maturity, pods per plant, protein content, and grain yield per plant in green gram.

Higher expected GAM coupled with high heritability of grain yield and its component traits like primary branches per plant(187.76) pods per plant(97.68), cluster per plant(74.54), seeds per pod (48.06) and pods per cluster(40.86). The higher GAM was also recorded for physiological traits governing drought tolerance such as leaf water potential (53.38), proline content (66.15), relative water content (41.13) and specific leaf area (56.43). Kousar et al., (2007) also reported high heritability coupled with genetic advance for pods per plant and plant height. Similar findings were reported by Gadakh et al., (2013) and Hemavathy et al., (2014)

The study revealed that germplasm accessions differed greatly for phenotypic coefficient of variation (PCV), genotypic coefficient of variation (GCV), heritability (broad sense) and genetic advancement for all the agronomic and physiological traits under drought condition. 


\section{Acknowledgement}

Dr. Kanavi, M.S.P., thanks Director of Research, University of Agricultural Sciences, Bangalore for giving financial assistance to carry out the research work

\section{References}

Ahmad, M., Zaffar, G., Razvi, S.M., Dar, Z.A., Mir, S.D., Bukhari, S.A. and Habib, M. 2014. Resilience of cereal crops to abiotic stress: a review. African J. Biotechnol., 13 (29): 2908292.

Allahmoradi, P., Ghobadi, M., Taherabadi, S. and Taherabadi, S. 2011. Physiological aspects of mung bean in response to drought stress. Singapore Int. Conf. Food Eng. Biotechnol., IPCBEE IACSIT Press, vol.9.

Baroowa, B. and Gogoi, N. 2015. Changes in plant water status, biochemical attributes and seed quality of black gram and green gram genotypes under drought. Int. lett. Nat. Sci., 42:1-12.

Beebe, S. E., Rao, I. M., Blair, M. W. and Acosta-Gallegos, J. A. 2013. Phenotyping common beans for adaptation to drought. Front. Physiol.,4(35): 1-19.

Fuller, D. Q. 2007. Contrasting patterns in crop domestication and domestication rates: recent archaeobotanical insights from the old world. Ann. Bot. (Lond.)., 100:903-924.

Gadakh, S.S., Dethe, A.M. and Kathale, M.N. 2013. Genetic variability, correlations and path analysis studies on yield and its components in mung bean (Vigna radiate L. Wilczek). Bioinfolet., 10(2A): 441-447.

Hanson, C. H., Robinson, H. F. and Comstock, R. E. 1956. Biometrical studies in yield in segregating populations of Korean lespediza.
Agron. J.,48: 214-318

Hemavathy, T. A., Shunmugavalli, N. and Anand, G. 2014. Genetic variability, correlation and path coefficient studies on yield and its components in mung bean (Vigna radiata L. Wilczek). Indian J. Agric. Res.,38(4): 442-446.

Johnson, H.W., Robinson, H.F. and Comstock, R.E. 1955. Estimation of genetic and environmental variability in soybeans. Agron. J.,47: 314-318

Kao, W. Y., Ehleringer, J. R. and Comstock, J. P. 1994. Variation in leaf movements among common bean cultivars. Crop sci.,34(5): 1273-1278.

Kate, A. M., Dahat, D. V. and Chavan, B.H. 2017. Genetic variability, heritability, correlation and path analysis studies in green gram (Vigna radiata L. Wilczek). Int. J. of Develop. Res.,7: 16704-16707.

Kousar, M., Abrahim, G., Jan, A. and Singh, K.A. 2007. Genetic variability and correlation studies on yield and its components in mung bean (Vigna radiata (L). Wilczek). J. Agron., 6(1): 216-218.

Muthuswamy, A. Jamunarani, M. and Ramakrishnan, P. 2019.Genetic variability, character association and path analysis studies in green gram (Vigna radiata (L.) Wilczek). Int. J. Curr. Microbiol. App. Sci., 8(4): 11361146.

Pandey, R.K., Herrera, W, AT., Villegas, AW., Penletion, JW. 1984. Drought response of grain legumes under irrigation gradient. III. plant growth. Agron. J., 76:557-560.

Pooja, Bangar., Ashok, Chaudhury., Bhavana, Tiwari., Sanjay Kumar., Ratna Kumari and Kangila, Venkataramana, Bhat. 2019. Morphophysiological and biochemical response of mung bean [Vigna radiata (L.) Wilczek] varieties at different developmental stages 
under drought stress.Turkish $J$. Biol.,43(1): 58-69.

Siddiqui, M.H, Oad, F.C., Buriro, U.A. 2007. Response of cotton cultivars to varying irrigation regimes. Asian $J$. Plant Sci.,6 (1):153-157.

Singh, V.P., Chhabra, A. and Kharb, R.P.S. 1988. Production and utilization of mung bean in India. Proc. Second Int. Symp. AVRDC, Shanhua, Bangkok, Thailand, pp:58.

Smartt, J. (1990). Grain legumes; evolution and genetic resources.Cambridge University Press: UK.

Tejbir, Singh., Amitesh, Sharma and Alie, F. A. 2009. Impact of environment on heritability and genetic gain for yield and its component traits in mung bean. Legume Res., 32(1): 55-58.

Yordanov, I., Velikova, V. and Tsone, V. 2000. Plant response to drought, acclimation and stress. Photosynthetica.,30:171-186.

\section{How to cite this article:}

Kanavi. M. S. P, G. Somu, N. Marappa, S. Rangaiah and Prakash Koler. 2020. Evaluation of Germplasm Accessions for Drought Tolerance in Green gram [Vigna radiata (L.)]. Int.J.Curr.Microbiol.App.Sci. 9(03): 1011-1024. doi: https://doi.org/10.20546/ijcmas.2020.903.119 